\title{
Effects of astrogaloside on the inflammation and immunity of renal failure patients receiving maintenance dialysis
}

\author{
RENLIAN SUN $^{1 *}$, HAIWEI REN ${ }^{1 *}$ and JIANXIN WEI ${ }^{2}$ \\ Departments of ${ }^{1}$ Nephrodialysis and ${ }^{2}$ Nephrology, Dezhou People's Hospital, Dezhou, Shandong 253014, P.R. China
}

Received August 1,2017; Accepted November 28, 2017

DOI: $10.3892 /$ etm.2018.5709

\begin{abstract}
Chronic renal failure is a type of clinical syndrome originating from chronic renal diseases. The aim of the study was to investigate the effect of astrogaloside on the inflammation and immunity of renal failure patients receiving maintenance dialysis. We randomly selected 92 renal failure patients receiving maintenance dialysis who were admitted to hospital for treatment between May, 2015 and April, 2016. Patients were randomly divided into the control $(n=46)$ and observation $(n=46)$ groups. Patients in the control group received the regular dialysis plus the basic treatment in Western medicine, while in the observation group, patients additionally received astrogaloside via intravenous injection as treatment. We compared the clinical efficacy of patients between the two groups, residual renal function (RRF), changes in urine volume, variations in inflammatory indicators [C-reaction protein (CRP), interleukin-6 (IL-6), IL-17, and tumor necrosis factor- $\alpha(\mathrm{TNF}-\alpha)$ ] before and after treatment, and the levels of the thymus-dependent lymphocyte (T cells) subgroup (CD3 ${ }^{+}$, $\mathrm{CD}^{+}, \mathrm{CD}^{+}$and $\left.\mathrm{CD}^{+} / \mathrm{CD}^{+}\right)$in the immune system of patients after treatment. In the observation group, the total effective rate was significantly higher than that in the control group $(\mathrm{P}<0.05)$. After 6 months, RRF and the urine volume of patients in the two groups were decreased when compared with the levels before treatment, and the decreasing rates of RRF and urine volume in the observation group were significantly lower than those in the control group $(\mathrm{P}<0.05)$. After treatment, the levels of human serum $C$ reaction protein (hs-CRP), IL-6, IL-17 and TNF- $\alpha$ in the two groups were lower than those before treatment, and the decrease in the observation group was more significant than that in the control group $(\mathrm{P}<0.05)$. Following treatment, the levels of $\mathrm{CD}^{+}, \mathrm{CD}^{+}$and $\mathrm{CD} 4^{+} / \mathrm{CD}^{+}$in the observation group were higher than those in the control group,
\end{abstract}

Correspondence to: Dr Jianxin Wei, Department of Nephrology, Dezhou People's Hospital, 1751 Xinhu Street, Dezhou, Shandong 253014, P.R. China

E-mail: ndsm22@126.com

*Contributed equally

Key words: astrogaloside, dialysis, renal failure, inflammation responses, immune functions and the level of $\mathrm{CD}^{+}$was lower than that in the control group $(\mathrm{P}<0.05)$. In conclusion, astrogaloside can delay the decrease in RRF of renal failure patients receiving the maintenance dialysis, ameliorate the inflammatory responses, and enhance the immune function, thereby increasing the disease resistance of patients and improving the clinical symptoms.

\section{Introduction}

Chronic renal failure is a kind of clinical syndrome originated from chronic renal diseases. China ranks in chronic renal disease, and according to a survey of epidemics, the incidence of chronic renal disease is more than $10 \%(1,2)$. Although the damaged renal function in renal failure patients cannot be reversed, effective ameliorations can be gained via effecatious treatment, which can prolong the survival time and improve the quality life of patients (3).

Currently, persistent dialysis remains the main method for the treatment of chronic renal failure. Nevertheless patients are susceptible to the complications of microinflammation, disorders in immune functions, and secondary infections after long-term dialysis. The combination treatment of traditional Chinese medicine (TCM) and Western medicine has been widely recognized as the major treatment, and the rational application of TCM combined with dialysis, not only delays the onset of renal failure and increases the efficacy of dialysis, but also decreases inflammation and enhances immune functions, thereby improving the life quality of patients $(4,5)$.

In the present study, renal failure patients undergoing dialysis received the combination therapy of Western medicine and treatment of astrogaloside, and the results can be considered as reliable evidence for improving the long-term survival rate and life quality of patients using this method.

\section{Materials and methods}

General material. We randomly selected 92 renal failure patients receiving maintenance dialysis who were admitted to the Dezhou Peaple's Hospital (Shandong, China) for treatment between May, 2015 and April, 2016. These patients were randomly divided into the control $(n=46)$ and observation $(n=46)$ groups. Inclusion criteria for the study were: i) Patients who met the criteria in the Clinical Practice Guidelines for Chronic Kidney Disease stipulated by the National Kidney Foundation (6); ii) patients who received regular dialysis 
Table I. General material of subjects.

\begin{tabular}{|c|c|c|c|c|}
\hline Item & Control group $(n=46)$ & Observation group $(n=46)$ & $\mathrm{t} / \chi^{2}$ & P-value \\
\hline Sex (male/female) & $26 / 20$ & $28 / 18$ & 0.179 & 0.672 \\
\hline Age (year) & $30-60$ & $30-65$ & & \\
\hline Average age (year) & $45.36 \pm 5.69$ & $46.85 \pm 5.78$ & 1.246 & 0.216 \\
\hline Disease course (month) & $52.62 \pm 7.46$ & $52.25 \pm 7.37$ & 0.239 & 0.811 \\
\hline Total cholesterol (mmol/l) & $5.72 \pm 0.84$ & $5.81 \pm 0.85$ & 0.511 & 0.611 \\
\hline Triglyceride (mmol/l) & $2.78 \pm 1.04$ & $2.82 \pm 0.83$ & 0.204 & 0.839 \\
\hline Body mass index (BMI, $\left.\mathrm{kg} / \mathrm{m}^{2}\right)$ & $23.43 \pm 3.27$ & $22.87 \pm 3.58$ & 0.783 & 0.435 \\
\hline Systolic pressure (mmHg) & $113.35 \pm 6.46$ & $112.94 \pm 6.35$ & 0.307 & 0.759 \\
\hline Diastolic pressure (mmHg) & $73.72 \pm 3.83$ & $74.34 \pm 4.76$ & 0.668 & 0.493 \\
\hline
\end{tabular}

for $>3$ months; iii) patients with a serum creatinine level of $<177 \mu \mathrm{mol} / 1$ and urine volume $\geq 200 \mathrm{ml}$; and iv) patients who signed the written informed consent. Exclusion criteria were: i) Patients who were complicated with severe diseases in heart, brain or liver, or with mental diseases; ii) patients with a medication history, such as glucocorticoid or cytotoxic drugs, or were in gestation or lactation period. Comparisons of general material between the two groups showed no statistically significant difference $(\mathrm{P}>0.05)$ (Table I).

Regular treatment. Pressures of patients in the two groups were strictly controlled using drugs, such as $\beta 2$ receptor blockers and calcium antagonist. Additionally, the diet of patients was under strict supervision, whereby patients received the high-quality protein diet according to the criteria of $0.8-1.2 \mathrm{~g} / \mathrm{kg} \cdot \mathrm{day}$, and corrections for electrolyte disturbance and imbalance between acid and base were carried out.

Hemodialysis for treatment. Patients in the two groups persistently received regular hemodialysis, using the hemodialysis machine manufactured by Braun Co., Ltd. (Melsungen, Germany). During hemodialysis, the volume of blood flow was set as $250 \mathrm{ml} / \mathrm{min}$, while the volume of reverse osmosis water and bicarbonate dialysing fluid was set as $500 \mathrm{ml} / \mathrm{min}$, and the polysulfone membrane was used as the dialysis membrane (area of 1.2-1.5 $\mathrm{m}^{2}$ ). Each week, the patients received hemodialysis three times ( $4 \mathrm{~h} / \mathrm{time})$ with the low-molecular-weight heparin as the anticoagulant.

Combination therapy of TCM and Western medicine. Patients in the control group only received the dialysis as treatment, while those in the observation group additionally received treatment using astrogaloside, in which Astragalus Injection (Shanghai Xinya Pharmaceutical Gaoyou Co., Ltd.; Chinese drug approval no. Z32021256) was administered via intravenous injection with the dose set as 10-20 ml/time and injection rate as $30 \mathrm{gtt} / \mathrm{min}$. Treatment lasted 6 months.

Detection of indexes. Before treatment and after 6 months since treatment, we detected the indexes of patients using the following apparatus: Flow cytometer (BD Biosciences, San Jose, CA, USA); automatic multifunctional microplate reader (Thermo Fisher Scientific, Shanghai, China); autobalancing centrifuge (Beijing Medical Centrifuge Factory, Beijing, China); and low-temperature refrigerator (Sanyo, Tokyo, Japan). The reagents used in this study included the hs-CRP, human serum IL-6, human serum IL-17 and human serum TNF- $\alpha$ kits, which were all produced by Adlitteram Diagnostic Laboratories (San Diego, CA, USA). Rabbit monoclonal antibodies of CD3 (cat no. ab16669), CD4 (cat no. ab133616) and CD8 (cat no. ab4055) (all from Shanghai Biotechnology Co., Ltd., Shanghai, China) were also used. Fasting venous blood $(3 \mathrm{ml})$ was drawn from patients in the two groups, and separated for the extraction of serum. CD3, CD4 and CD8 antibodies were then added into the serum for $30 \mathrm{~min}$ of incubation at $4^{\circ} \mathrm{C}$ in the dark, and a flow cytometer (BD Biosciences) was used to detect and calculate the $\mathrm{CD}^{+} / \mathrm{CD}^{+}$ratio. Detection was carried out via an enzymelinked immunosorbent assay (ELISA) in strict accordance with the instructions of the hs-CRP, IL-6, IL-17 and TNF- $\alpha$ kits. After $15 \mathrm{~min}$ of incubation at $20^{\circ} \mathrm{C}$ in the dark, $50 \mu \mathrm{l}$ stop buffer was added followed by reading the optical density (OD) value at a wavelength of $450 \mathrm{~nm}$ for $15 \mathrm{~min}$ using the microplate reader. Concentrations of hs-CRP, IL-6, IL-17 and TNF- $\alpha$ were then calculated.

Evaluation indexes. Using the TCM symptom integral, the symptoms of patients including insufficiency of qi and strength, lusterless complexion, loss of appetite, and anorexia, were assessed according to the following criteria: i) 0 point, no such symptom; ii) 1 point, occasionally but not affecting regular life and work; iii) 2 points, frequently, and slightly affecting regular life and work; iv) 3 points, permanently, and severely affecting regular life and work. Efficacy standard: i) Cured: After treatment, all the symptoms disappeared with a decreased rate of symptom integral of $>90 \%$; ii) excellently effective: After treatment, most of the symptoms disappeared with a decreased rate of symptom integral of $>70 \%$; iii) effective: After treatment, some of the symptoms disappeared with a decreased rate of symptom integral of $>30 \%$; iv) invalid: After treatment, no improvement or aggravation was seen in clinical symptoms with a decreased rate of symptom integral of $<30 \%$.

Variations of RRF and urine volume of patients in both groups before and after treatment, were detected and calculated as: $\mathrm{RRF}=[$ (concentration of urea nitrogen in urine/concentration of urea nitrogen in serum); urine volume in $24 \mathrm{~h} / 1440+$ (concentration of creatinine in urine/concentration of creatinine in serum); urine volume in $24 \mathrm{~h} / 1440 \mathrm{~J} / 2$. A decreased rate of RRF was calculated as (RRF before 
Table II. Comparison of TCM symptom integral of patients between the two groups.

\begin{tabular}{lcccr}
\hline & & \multicolumn{3}{c}{ TCM symptom integral } \\
\cline { 3 - 5 } Group & Case & Before treatment & After treatment & t-value \\
\hline Observation group & 46 & $7.36 \pm 1.22$ & $3.34 \pm 0.82$ & 18.548 \\
Control group & 46 & $7.35 \pm 1.33$ & $4.48 \pm 1.27$ & 10.585 \\
t-value & & 0.376 & 5.115 & $<0.001$ \\
P-value & & 0.970 & $<0.001$ & \\
\hline
\end{tabular}

TCM, traditional Chinese medicine.

Table III. Comparison of efficacy of patients between the two groups (n, \%).

\begin{tabular}{lrrrr}
\hline Group & $\mathrm{N}$ & Cured & Excellently effective & Effective \\
\hline Observation group & 46 & $21(38.88)$ & $10(30.55)$ & $9(19.44)$ \\
Control group & 46 & $9(16.66)$ & $7(22.22)$ & $6(13.04)$ \\
\hline
\end{tabular}

After the ranked sum test, comparison on the efficacy of patients between the two groups shows $\mathrm{Z}=3.39$ and $\mathrm{P}=0.001<0.05$.

Table IV. Comparisons on RRF and urine volume of patients in the two groups before and after treatment.

\begin{tabular}{|c|c|c|c|c|c|c|c|c|}
\hline \multirow[b]{2}{*}{ Group } & \multicolumn{4}{|c|}{$\mathrm{RRF}$ (ml/min) } & \multicolumn{4}{|c|}{ Urine volume (ml) } \\
\hline & $\begin{array}{c}\text { Before } \\
\text { treatment }\end{array}$ & $\begin{array}{c}\text { After } \\
\text { treatment }\end{array}$ & t-value & P-value & $\begin{array}{c}\text { Before } \\
\text { treatment }\end{array}$ & After treatment & t-value & P-value \\
\hline Observation group & $2.13 \pm 1.32$ & $1.14 \pm 0.98$ & 4.084 & $<0.001$ & $589.64 \pm 21.73$ & $455.41 \pm 11.62$ & 36.945 & $<0.001$ \\
\hline Control group & $2.12 \pm 1.28$ & $0.58 \pm 0.57$ & 7.454 & $<0.001$ & $590.56 \pm 22.64$ & $317.35 \pm 11.45$ & 73.037 & $<0.001$ \\
\hline t-value & 0.037 & 6.940 & & & 0.199 & 57.399 & & \\
\hline P-value & 0.971 & $<0.001$ & & & 0.843 & $<0.001$ & & \\
\hline
\end{tabular}

RRF, residual renal function.

treatment - RRF after treatment)/observation duration. A decreased rate of urine volume was calculated as: (urine volume before treatment - urine volume after treatment)/ observation duration.

Prior to treatment and 6 months after treatment, 3-5 ml fasting venous blood was collected from patients in the two groups to extract the serum. The levels of hs-CRP, IL-6, IL-17 and TNF- $\alpha$ in serum of patients were assayed via ELISA. Six months after treatment, the levels of T-lymphocyte subgroups $\mathrm{CD}^{+}, \mathrm{CD}^{+}, \mathrm{CD}^{+}$and $\mathrm{CD} 4^{+} / \mathrm{CD}^{+}$were detected using a flow cytometer (BD Biosciences).

Statistical analysis. Statistical package for social science 19.0 (SPSS; SPSS Inc., Chicago, IL, USA) software was used for data processing. Measurement data were presented by mean \pm standard deviation (mean $\pm \mathrm{SD}$ ), Chi-squared test and the $\mathrm{t}$-test were used for comparison of data. $\mathrm{P}<0.05$ suggested that the difference had statistical significance.

\section{Results}

Comparison of TCM symptom integral and efficacy of patients between the two groups. After treatment, the TCM symptom integral of patients in the observation group was $(3.34 \pm 0.82)$, which was significantly lower than that of the control group $(4.48 \pm 1.27)$. The total effective rate of the observation group was $86.96 \%$, which was significantly higher than that in the control group (60.87\%). Differences in comparisons were statistically significant $(\mathrm{P}<0.05)$ (Tables II and III).

Comparisons on RRF and urine volume of patients in the two groups before and after treatment. After treatment, RRF and urine volume of patients in the two groups were decreased when compared with the levels before treatment, and the differences were statistically significant $(\mathrm{P}<0.05)$. Differences in the comparisons of RRF and urine volume of patients between the two groups showed statistical significance 


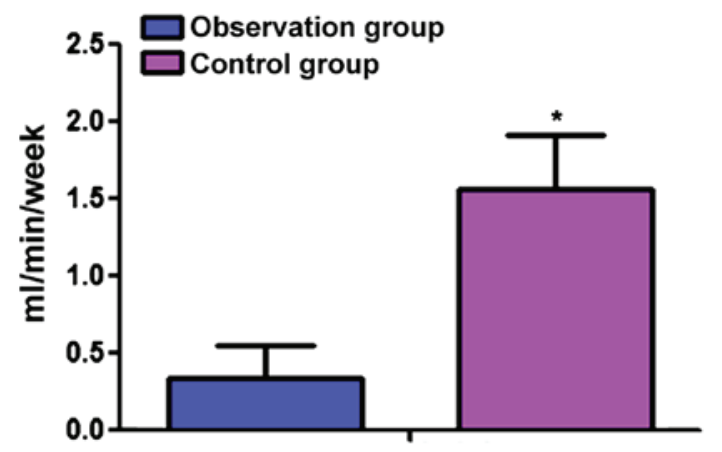

Figure 1. Decreased rates in residual renal function (RRF) of patients of the two groups after treatment. Comparisons between the observation and the control groups. ${ }^{*} \mathrm{P}<0.05$, statistically significant.

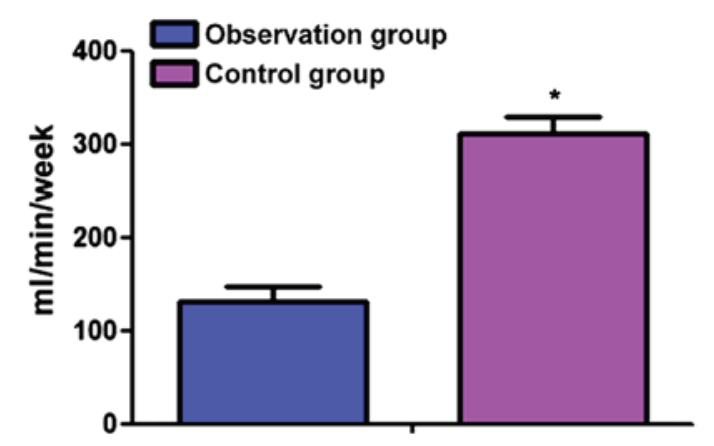

Figure 2. Decreased rates in urine volume in patients of the two groups after treatment. Comparisons between the observation and control groups. ${ }^{*} \mathrm{P}<0.05$, statistically significant.

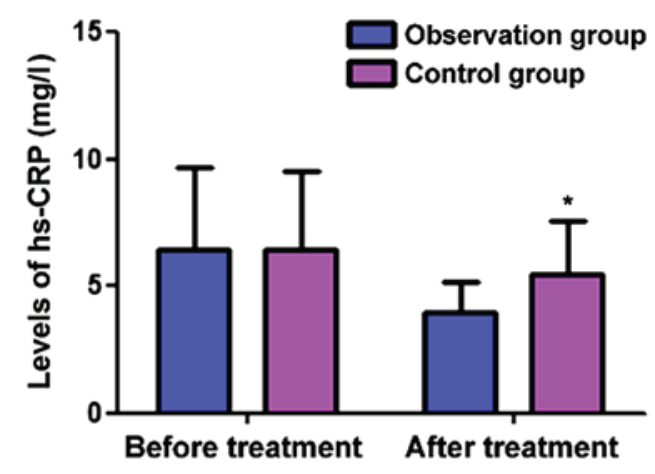

Figure 3. Levels of human serum $\mathrm{C}$ reaction protein (hs-CRP) of patients in both groups. Comparison between the two groups before treatment shows $\mathrm{P}>0.05$. Comparisons before and after treatment in the two groups, and between the two groups after treatment. ${ }^{*} \mathrm{P}<0.05$, statistically significant.

$(\mathrm{P}<0.05)$. In the observation group, the decreased rate of RRF was $0.34 \pm 0.23 \mathrm{ml} / \mathrm{min} /$ week, and that of urine volume was $132.25 \pm 6.13 \mathrm{ml} /$ week, while for patients in the control group, the decreased rate of RRF was $1.57 \pm 0.83 \mathrm{ml} / \mathrm{min} /$ week, and that of urine volume was $312.34 \pm 8.54 \mathrm{ml} /$ week. Decreased rates in RRF and urine volume of patients in the observation group were lower than those in the control, and the differences were statistically significant $(\mathrm{P}<0.05)$ (Table IV; Figs. 1 and 2).

Comparisons of levels of hs-CRP, IL-6, IL-17 and TNF- $\alpha$ of patients between the two groups. Prior to and after

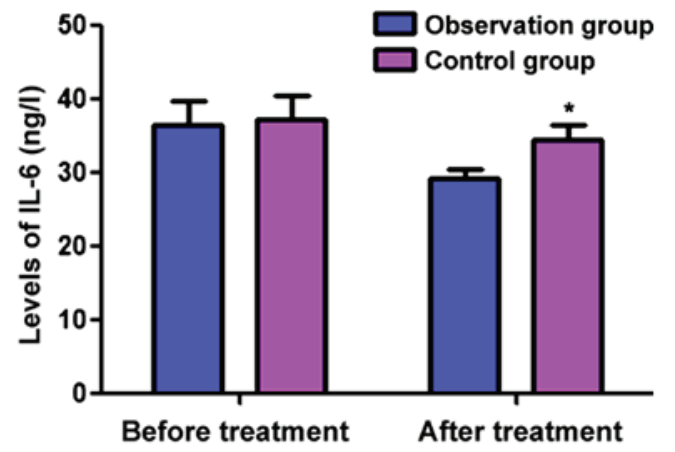

Figure 4. Levels of interleukin-6 (IL-6) of patients in the two groups. Comparison of the two groups before treatment shows no significant difference $(\mathrm{P}>0.05)$. Comparisons before and after treatment in the two groups, and between the two groups after treatment. ${ }^{*} \mathrm{P}<0.05$, statistically significant.

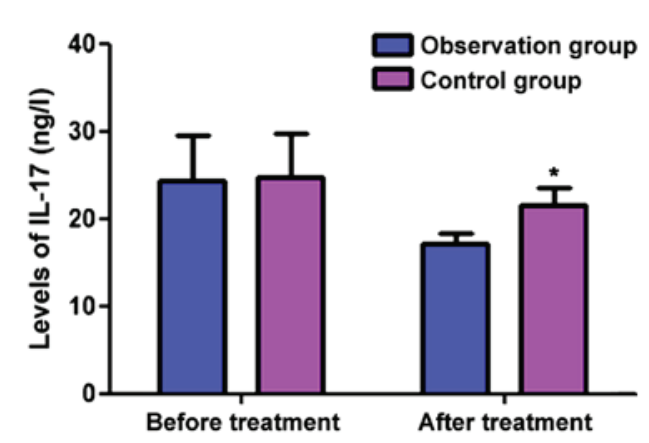

Figure 5. Levels of interleukin-17 (IL-17) of patients in both groups: Comparison between the two groups before treatment shows $\mathrm{P}>0.05$; comparisons before and after treatment in the two groups, and between the two groups after treatment. ${ }^{*} \mathrm{P}<0.05$, statistically significant.

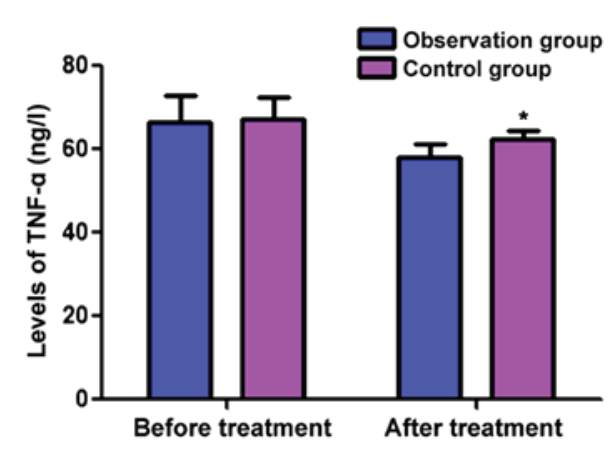

Figure 6. Levels of tumor necrosis factor- $\alpha$ (TNF- $\alpha)$ of patients in both groups. Comparisons between the two groups before treatment show $\mathrm{P}>0.05$. Comparisons before and after treatment in the two groups, and between the two groups after treatment. ${ }^{*} \mathrm{P}<0.05$, statistically significant.

6 months since treatment, the levels of hs-CRP, IL-6, IL-17 and TNF- $\alpha$ in the observation group were, respectively, $6.43 \pm 3.24$ vs. $3.67 \pm 1.23 \mathrm{mg} / 1,36.43 \pm 4.24$ vs. $29.67 \pm 3.23 \mathrm{ng} / 1$, $24.43 \pm 6.24$ vs. $17.67 \pm 3.23 \mathrm{ng} / 1$, and $66.43 \pm 5.24$ vs. $57.67 \pm 3.23 \mathrm{ng} / \mathrm{l}$. Before and after 6 months since treatment, the levels of hs-CRP, IL-6, IL-17 and TNF- $\alpha$ in the control group were, respectively, $6.65 \pm 3.35$ vs. $5.63 \pm 2.15 \mathrm{mg} / 1$, $37.24 \pm 4.36$ vs. $34.58 \pm 3.74 \mathrm{ng} / 1,24.68 \pm 6.58$ vs. $21.67 \pm 3.23 \mathrm{ng} / 1$ and $67.36 \pm 5.17$ vs. $62.85 \pm 4.43 \mathrm{ng} / \mathrm{l}$. After treatment, the levels of CRP, IL-6, IL-17 and TNF- $\alpha$ of patients in the two groups were significantly decreased compared to the levels before 
Table V. Comparisons of T-cell subgroup levels between the two groups after 6 months since treatment.

\begin{tabular}{lccccc}
\hline Item & Case & $\mathrm{CD}^{+}$ & $\mathrm{CD}^{+}$ & $\mathrm{CD}^{+}$ & $\mathrm{CD}^{+} / \mathrm{CD}^{+}$ \\
\hline Observation group & 46 & $71.52 \pm 6.73$ & $42.75 \pm 7.26$ & $23.47 \pm 6.35$ & $1.83 \pm 0.56$ \\
Control group & 46 & $64.36 \pm 6.42$ & $37.43 \pm 7.63$ & $27.22 \pm 6.15$ & $1.45 \pm 0.53$ \\
t-value & & 5.221 & 3.426 & 2.877 & 3.343 \\
P-value & $<0.001$ & 0.001 & 0.005 & 0.001 \\
\hline
\end{tabular}

treatment, and at different time points, decreases in the observation group, were more significant than those in the control group $(\mathrm{P}<0.05)($ Figs. 3-6)

Comparisons of T-cell subgroup levels between the two groups after 6 months since treatment. The levels of $\mathrm{CD}^{+}, \mathrm{CD}^{+}$and $\mathrm{CD}^{+} / \mathrm{CD}^{+}$of patients in the observation group were higher than those in the control group, and the level of $\mathrm{CD}^{+}$was lower than that in the control group $(\mathrm{P}<0.05)($ Table $\mathrm{V})$.

\section{Discussion}

General profile of renal failure. The disease course of chronic renal failure is generally characterized by irreversibility, progressive development and aggravation, which can be divided into 4 phases: renal insufficiency compensation phase, decompensation phase of chronic renal failure, renal failure phase, and end-phase of renal failure (i.e., the phase of uremia) (7). At present, the pathogenesis of chronic renal failure remains unclear, and the potential pathogenesis in kidney is injured by various pathogens, resulting in the loss of regular functions in some nephrons. Thus, remaining normal nephrons should compensate for the loss in functions of normal nephrons to meet regular demand by working intensively. At the same time, renal tubules experience continuous dilation, resulting in an increase in the volume of crude urine with an increse of the load in reabsorption. Renal damage, once persistently progressing to a certain degree, may lead to continuous decrease in renal functions, which causes glomerular hypertrophy and platelet coagulation, thus inducing the formation of microthrombus and facilitating glomerular sclerosis. Consequently, glomerular permeability increases at an increment in proteinuria, which can further damage the tubulointerstitium. Such a vicious circle leads to excacerbation of the symptoms of renal failure $(8,9)$. Results of TCM, suggest renal failure is caused by internal and external factors, with internal factors refering to qi of kidney, while external factors refer to the 'evil' of pain and toxin, exogenous diseases caused by six excessive atmospheric factors and nephrotoxic drugs. Deficiency in qi of kidney, plus the evil of pain and toxin, and the invasion of six external excessive atmospheric factors, and irregular excessive administration of nephrotoxic drugs, contribute to renal failure (10).

Treatment of renal failure. In Western medicine, treatment methods include limitations in intake of protein and calorically dense food, controlling high pressure within glomerulus and systemic hypertension via dialysis, supplementation of amino acid and renal transplantation (11). Through considerable clinical data, it has been confirmed that the life of patients can be prolonged through persistent and rational dialysis, and most patients can survive for over 20 years (12). Despite the promising efficacy, renal transplantation is bound by difficulties involved in finding an appropriate source of kidney. In addition, renal transplantation results in financial burden on the patients and their family, leading to more obstacles in spreading and promoting the application of renal transplantation (13). However, in TCM treatment, progression in renal failure is delayed mainly by maintaining the essence of nephron (14). In this study, promising efficacy was obtained through astrogaloside medication for patients, where in the observation group, the TCM symptom integral was significantly lower than that in the control group, and the total effective rate was significantly higher than that in the control group $(\mathrm{P}<0.05)$. This difference occured because astrogaloside injection, which is prepared through the extractions of astrogalus has the effects of antiperspirant, detoxification, replenishment of qi, consolidation of exterior, diuresis, tissue regeneration and detumescence, which can effectively inhibit the aggregation of tubulointerstitial extracellular matrix, improve the microcirculation, increase the blood flow volume in kidney and protect the endothelium, thus enhancing the efficacy of dialysis and delaying progression of the disease $(15,16)$. The results of this study have shown that after 6 months since treatment, the decrease rates in RRF and urine volume of patients in the observation group were lower than those in the control group $(\mathrm{P}<0.05)$. This reduction is because the decrease in RRF is caused by gradual asthenia in qi of kidney according to TCM, while astrogaloside can supplement the acquired qi of the body, thereby nourishing the congenital qi, to reach the purpose of invigorating the qi of kidney. In addition, TCM believes that qi can govern the blood, and astrogaloside can supplement the qi of kidney to prevent the loss of nutrient substances in hemodialysis, and improve the glomerular filtrating barrier to protect the residual urine of patients (17).

Effect of astrogaloside on inflammation and immune functions. In renal failure patients, conditions, such as proteinuria and hyperactivity of the renin-angiotensin system, can activate in vivo inflammatory responses, and inhibition of the immune functions of T lymphocytes, resulting in the disorders in immune functions (18). Results of the present study indicated that after 6 months since treatment, the levels of hs-CRP, IL-6, IL-17 and TNF- $\alpha$ of patients in the two groups were significantly decreased when compared with the levels before treatment, and the decrease in the observation group was more significant than that in the control group $(\mathrm{P}<0.05)$. $\mathrm{CD}^{+}, \mathrm{CD}^{+}{ }^{+}$and $\mathrm{CD}^{+} / \mathrm{CD}^{+}$levels in the observation group 
were higher than those in the control group, and the $\mathrm{CD}^{+}$level was lower than that in the control group $(\mathrm{P}<0.05)$ because $\mathrm{CD}^{+}$, a subgroup of the inductive $\mathrm{T}$ cell, has a positive effect on immune regulation, while astrogaloside can modulate the disorder in immune functions, thus reducing the transformation of $\mathrm{CD}^{+}$to Th17 cells and the release of inflammatory factors in serum, such as IL-17. CD8 ${ }^{+}$belongs to a subgroup of inhibitory $\mathrm{T}$ cells, and under the activated state, it can destroy and kill the affected cells, and inhibit the activity of $\mathrm{CD}^{+}$, which can cause disorder in the immune functions. Thus, the $\mathrm{CD}^{+} / \mathrm{CD}^{+}$ratio can reflect the status of cellular immune and immune responses of the body to a certain degree (19). The disorder of immune functions can induce the persistent activation of Th17 cells, resulting in an excessive release of IL-17, which can activate the nuclear factor $-\kappa \mathrm{B}(\mathrm{NF}-\kappa \mathrm{B})$ signal pathway of the resident cells in kidney. NF- $\kappa \mathrm{B}$ can modulate the activities of hs-CRP, IL-6, IL-17 and TNF- $\alpha$ in inflammatory responses, which further induce high expressions to aggravate the inflammatory responses and accelerate the progress of renal failure (20). Astrogaloside has distinct regulatory effects on humoral immune and non-specific immune, and the effective anti-inflammation capability.

In conclusion, astrogaloside can protect renal functions, prevent renal fibrosis, delay the progression of chronic renal failure, effectively ameliorate the status of inflammatory responses, and modulate the immune functions of patients.

\section{References}

1. Zhang L, Wang F, Wang L, Wang W, Liu B, Liu J, Chen M, He Q, Liao Y, Yu X, et al: Prevalence of chronic kidney disease in China: A cross-sectional survey. Lancet 379: 815-822, 2012.

2. Chawla LS, Eggers PW, Star RA and Kimmel PL: Acute kidney injury and chronic kidney disease as interconnected syndromes. N Engl J Med 371: 58-66, 2014.

3. Carville S, Wonderling D and Stevens P; Guideline Development Group: Early identification and management of chronic kidney disease in adults: Summary of updated NICE guidance. BMJ 349: g4507, 2014.

4. Guan Y, Wu XX, Duan JL, Yin Y, Guo C, Wei G, Wang YH, Zhu YR, Weng Y, Xi MM, et al: Effects and mechanism of combination of Rhein and Danshensu in the treatment of chronic kidney disease. Am J Chin Med 43: 1381-1400, 2015.

5. Teschke R, Zhang L, Long H, Schwarzenboeck A, SchmidtTaenzer W, Genthner A, Wolff A, Frenzel C, Schulze J and Eickhoff A: Traditional Chinese medicine and herbal hepatotoxicity: A tabular compilation of reported cases. Ann Hepatol 14: 7-19, 2015.

6. KDOQI: KDOQI Clinical Practice Guidelines and Clinical Practice Recommendations for Diabetes and Chronic Kidney Disease. Am J Kidney Dis 49: 12-154, 2007.

7. Gámez-Méndez AM, Vargas-Robles H, Arellano-Mendoza M, Cruz-Laguna E, Rios A and Escalante B: Early stage of obesity potentiates nitric oxide reduction during the development of renal failure. J Nephrol 27: 281-287, 2014.
8. Ninomiya T, Perkovic V, Turnbull F, Neal B, Barzi F, Cass A, Baigent C, Chalmers J, Li N, Woodward M, et al; Blood Pressure Lowering Treatment Trialists' Collaboration: Blood pressure lowering and major cardiovascular events in people with and without chronic kidney disease: Meta-analysis of randomised controlled trials. BMJ 347: f5680, 2013.

9. Huang SH, Filler G and Lindsay RM: Residual renal function calculated from serum cystatin $\mathrm{C}$ measurements and knowledge of the weekly standard Kt/V urea. Perit Dial Int 32: 102-104, 2012.

10. Li JY, Yu JS, Hu WH, Du YJ and Liu XY: Study on the characteristics of syndrome distribution of malnutrition in chronic renal failure in traditional Chinese medicine. World J Integr Trad West Med 724-726, 2009.

11. Coresh J and Jafar TH: Disparities in worldwide treatment of kidney failure. Lancet 385: 1926-1928, 2015.

12. Nistor I, Palmer SC, Craig JC, Saglimbene V, Vecchio M, Covic A and Strippoli GF: Convective versus diffusive dialysis therapies for chronic kidney failure: An updated systematic review of randomized controlled trials. Am J Kidney Dis 63: 954-967, 2014.

13. Pihlstrøm H, Mjøen G, Dahle DO, Pilz S, Midtvedt K, März W, Abedini S, Holme I, Fellström B, Jardine A, et al: Symmetric dimethylarginine as predictor of graft loss and all-cause mortality in renal transplant recipients. Transplantation 98: 1219-1225, 2014.

14. Gao H, Wang T and Yu RH: A cohort study on delaying the progress of chronic renal failure mainly with Modified Shenqi Dihuang Decoction. Zhongguo Zhong Xi Yi Jie He Za Zhi 32: 39-42, 2012 (In Chinese).

15. Lv L, Wu SY, Wang GF, Zhang JJ, Pang JX, Liu ZQ, Xu W, Wu SG and Rao JJ: Effect of astragaloside IV on hepatic glucoseregulating enzymes in diabetic mice induced by a high-fat diet and streptozotocin. Phytother Res 24: 219-224, 2010.

16. Gui D, Guo Y, Wang F, Liu W, Chen J, Chen Y, Huang J and Wang N: Astragaloside IV, a novel antioxidant, prevents glucoseinduced podocyte apoptosis in vitro and in vivo. PLoS One 7: e39824, 2012

17. Qi W, Niu J, Qin Q, Qiao Z and Gu Y: Astragaloside IV attenuates glycated albumin-induced epithelial-to-mesenchymal transition by inhibiting oxidative stress in renal proximal tubular cells. Cell Stress Chaperones 19: 105-114, 2014.

18. Norouzi J, Yadollahpour A, Mirbagheri SA, Mazdeh MM and Hosseini SA: Predicting renal failure progression in chronic kidney disease using integrated intelligent fuzzy expert system. Comput Math Methods Med 2016: 6080814, 2016.

19. Moutaftsi M, Tscharke DC, Vaughan K, Koelle DM, Stern L, Calvo-Calle M, Ennis F, Terajima M, Sutter G, Crotty S, et al: Uncovering the interplay between CD8, CD4 and antibody responses to complex pathogens. Future Microbiol 5: 221-239, 2010.

20. Noack M and Miossec P: Th17 and regulatory T cell balance in autoimmune and inflammatory diseases. Autoimmun Rev 13: 668-677, 2014. 